\title{
GLOBAL GRADIENT ESTIMATES IN WEIGHTED LEBESGUE SPACES FOR PARABOLIC OPERATORS
}

\author{
Sun-Sig Byun, Dian K. Palagachev and Lubomira G. Softova \\ Seoul National University, Department of Mathematical Sciences and \\ Research Institute of Mathematics, Seoul 151-747, Korea; byun@snu.ac.kr \\ Politecnico di Bari, Dipartimento di Meccanica, Matematica e Management (DMMM) \\ Via E. Orabona 4, 70125 Bari, Italy; dian.palagachev@poliba.it \\ Second University of Naples, Department of Civil Engineering, Design, Construction \\ Industry and Environment, Via Roma 29, 81031 Aversa, Italy; luba.softova@unina2.it
}

\begin{abstract}
We deal with the regularity problem for linear, second order parabolic equations and systems in divergence form with measurable data over non-smooth domains, related to variational problems arising in the modeling of composite materials and in the mechanics of membranes and films of simple non-homogeneous materials which form a linear laminated medium. Assuming partial BMO smallness of the coefficients and Reifenberg flatness of the boundary of the underlying domain, we develop a Calderón-Zygmund type theory for such parabolic operators in the settings of the weighted Lebesgue spaces. As consequence of the main result, we get regularity in parabolic Morrey scales for the spatial gradient of the weak solutions to the problems considered.
\end{abstract}

\section{Introduction}

The general aim of the present article is to develop a weighted $L^{p}$-CalderónZygmund type theory for divergence form, linear parabolic systems with discontinuous coefficients over domains with rough boundary. More precisely, we characterize the regularity of the weak solutions to such systems by deriving global estimates for the spatial gradient in the framework of the weighted Lebesgue spaces, generalizing this way the recent unweighted $L^{p}$-results of Byun $[1,2]$, Byun, Palagachev and Wang [5], Byun and Wang [6], Dong [11], Dong and Kim [12], and Palagachev and Softova [24]

Let $\Omega \subset \mathbf{R}^{n}$ be a bounded domain, $n \geq 2$, and set $Q=\Omega \times(0, T]$ for the cylinder in $\mathbf{R}^{n+1}$ with base $\Omega$ and of height $T$. We consider the following Cauchy-Dirichlet problem

$$
\begin{cases}u_{t}-D_{\alpha}\left(a^{\alpha \beta}(x, t) D_{\beta} u\right)=D_{\alpha} f^{\alpha}(x, t) & \text { in } Q, \\ u(x, t)=0 & \text { on } \partial_{P} Q,\end{cases}
$$

where $\partial_{P} Q=\partial \Omega \times[0, T] \cup \Omega \times\{t=0\}$ stands for the parabolic boundary of $Q$ and the summation convention over the repeated indices, running from 1 to $n$, is understood.

Suppose that the coefficient matrix $\mathbf{a}(x, t)=\left\{a^{\alpha \beta}(x, t)\right\}_{\alpha, \beta=1}^{n}: \mathbf{R}^{n+1} \rightarrow \mathbf{M}^{n \times n}$ is measurable, uniformly bounded and uniformly parabolic, that is, there exist positive

doi:10.5186/aasfm.2016.4102

2010 Mathematics Subject Classification: Primary 35K20; Secondary 35R05, 35B65, 35B45, 46E30, 35K40, 42B25, 74E30.

Key words: Weighted Lebesgue space, Muckenhoupt weight, parabolic system, CauchyDirichlet problem, measurable coefficients, BMO, gradient estimates, Morrey space, linear laminates. 
constants $L$ and $\nu$ such that

$$
\left\{\begin{array}{l}
\left\|a^{\alpha \beta}\right\|_{L^{\infty}\left(\mathbf{R}^{n+1}\right)} \leq L, \\
a^{\alpha \beta}(x, t) \xi_{\alpha} \xi_{\beta} \geq \nu|\xi|^{2} \quad \forall \xi \in \mathbf{R}^{n}, \text { for almost all }(x, t) \in \mathbf{R}^{n+1} .
\end{array}\right.
$$

Denote the non-homogeneous term in (1.1) by $\mathbf{F}(x, t)=\left(f^{1}(x, t), \ldots, f^{n}(x, t)\right)$. It is well known (cf. $[2,6]$ and the references therein) that if $\mathbf{F} \in L^{2}(Q)$ then the problem (1.1) has a unique weak solution. Recall that a weak solution of this problem is a function

$$
u \in C^{0}\left(0, T ; L^{2}(\Omega)\right) \cap L^{2}\left(0, T ; H_{0}^{1}(\Omega)\right)
$$

that satisfies

$$
\int_{Q} u \varphi_{t} d x d t-\int_{Q} a^{\alpha \beta} D_{\beta} u D_{\alpha} \varphi d x d t=\int_{Q} f^{\alpha} D_{\alpha} \varphi d x d t
$$

for all $\varphi \in C_{0}^{\infty}(Q)$ with $\varphi(x, T)=0$. Moreover, the following $L^{2}$-estimate holds

$$
\int_{Q}|D u|^{2} d x d t \leq c \int_{Q}|\mathbf{F}|^{2} d x d t
$$

where the constant $c$ depends only on $n, L, \nu$ and $T$.

A natural extension of (1.3) would be the estimate

$$
\int_{Q}|D u|^{p} d x d t \leq c \int_{Q}|\mathbf{F}|^{p} d x d t
$$

with $p>1$ or, more generally,

$$
\int_{Q}|D u|^{p} \omega(x, t) d x d t \leq c \int_{Q}|\mathbf{F}|^{p} \omega(x, t) d x d t
$$

with suitable conditions imposed on the exponent $p$ and the weight $\omega(x, t)$.

Indeed, the sole parabolicity of the differential operator considered and boundedness of the underlying domain $\Omega$ are not enough to ensure the validity of (1.4) in general. In order to have (1.4) for the weak solution to any system (1.1), one has to impose some regularity requirements on the coefficient matrix $\mathbf{a}$, some finer geometric assumption on $\partial \Omega$ and suitable conditions on the weight function $\omega$.

What is our main concern in the present paper is to indicate that set of essentially optimal hypotheses on the data of (1.1) which ensure (1.4), and to develop a Calderón-Zygmund type theory for the problem under consideration. Namely, taking the non-homogeneous term $\mathbf{F}$ in the weighted Lebesgue space $L_{\omega}^{p}(Q)$ (see Sections 2 and 3 for the corresponding definitions) we prove that the spatial gradient $D u$ of the weak solution $u$ to (1.1) belongs to the same space $L_{\omega}^{p}(Q)$, what is actually the estimate (1.4).

Restricting the value of the exponent $p$ in the range $(2, \infty)$, we consider weights $\omega(x, t)$ belonging to the parabolic Muckenhoupt class $A_{\frac{p}{2}}$. This is a necessary and sufficient restriction ensuring boundedness of the Hardy-Littlewood maximal operator when acting on the weighted Lebesgue spaces $L_{\omega}^{p}$. For what concerns the coefficients $a^{\alpha \beta}(x, t)$ of the operator considered, we suppose these are only measurable with respect to one spatial variable and are averaged in the sense of small bounded mean oscillation (BMO) in the remaining space and time variables. This partially BMO assumption on the coefficients is quite general and allows arbitrary discontinuity in one spatial direction which is often related to problems of linear laminates, while the behaviour with respect to the other directions, including the time, are controlled in terms of small-BMO, such as small multipliers of the Heaviside step function for 
instance. It is clear that the cases of continuous, VMO or small-BMO principal coefficients with respect to all variables are particular cases of the situation here considered. Regarding the underlying domain $\Omega$, we suppose that its non-smooth boundary is Reifenberg flat (cf. [23]) that means $\partial \Omega$ is well approximated by hyperplanes at each point and at each scale. This is a sort of minimal regularity of the boundary, guaranteeing validity in $\Omega$ of some natural properties of geometric analysis and partial differential equations such as $W^{1, p}$-extension, non-tangential accessibility property, measure density condition, the Poincaré inequality and so on. We refer the reader to $[10,17,18,30]$ and the references therein for further details. In particular, a domain which is sufficiently flat in the sense of Reifenberg is also Jones flat. Moreover, domains with $C^{1}$-smooth or Lipschitz continuous boundaries with small Lipschitz constant belong to that category, but the class of Reifenberg flat domains extends beyond these common examples and contains domains with rough fractal boundaries such as the Helge von Koch snowflake.

It is worth noting that the weighted $L^{p}$-regularity theory here developed is related to important variational problems arising in modeling of deformations in composite materials as fiber-reinforced media or, more generally, in the mechanics of membranes and films of simple non-homogeneous materials which form a linear laminated medium. In particular, a highly twinned elastic or ferroelectric crystal is a typical situation where a laminate appears. The equilibrium equations of such a linear laminate usually have only bounded and measurable coefficients in the direction of the stratification. We refer the reader to the seminal papers $[8,20,19]$ for the general statement of the problem and various issues regarding regularity of solutions in case of piecewise smooth coefficients, and to the more recent works $[13,11]$ for further developments. The non-smoothness of the underlying Reifenberg flat domain, instead, is related to models of real-world systems over media with fractal geometry such as blood vessels, the internal structure of lungs, bacteria growth, graphs of stock market data, clouds, semiconductor devices, etc.

The paper is organized as follows. Section 2 collects some auxiliary results from the harmonic analysis regarding the properties of the Muckenhoupt weights and boundedness of the Hardy-Littlewood maximal operator on the weighted Lebesgue spaces, while in Section 3 we set down the hypotheses on the data of problem (1.1) and state the main result of the paper (Theorem 3.2). The estimate in the weighted Lebesgue spaces $L_{\omega}^{p}$ of the spatial gradient $D u$ of the weak solution to (1.1) is proved in Section 4. The main analytic tools employed in that proof rely on the Vitali covering lemma, boundedness properties of the Hardy-Littlewood maximal operator on weighted spaces, power decay estimates of the upper level sets of the spatial gradient and fine properties of the Muckenhoupt weights $\omega(x, t)$. As an outgrowth of the main result, we show in Section 5 that the Calderón-Zygmund property still holds true in the framework of the parabolic Morrey scales $L^{p, \lambda}$ by showing that $\mathbf{F} \in L^{p, \lambda}$ yields $D u \in L^{p, \lambda}$. A crucial step of our approach here is ensured by an old result of Coifman and Rochberg [9] ensuring that a suitable power of the maximal operator of a characteristic function is an $A_{1}$-weight. Without essential difficulties, the technique employed in proving regularity of solution to the equation in (1.1) could be extended to the case of systems and, that is why, in the final Section 6 we restrict ourselves to announce only the weighted $L^{p}$-regularity result for the weak solutions to linear, second order parabolic systems with partially BMO coefficients over Reifenberg flat domains. 
To this end, let us note that in the case of elliptic equations, weighted $L^{p_{-}}$ regularity results have been proved in [21] under the small-BMO assumption with respect to all variables, and in [3, 4] for equations with partially BMO coefficients. To the best of our knowledge, the results here obtained are the first of this kind in the settings of parabolic weighted spaces.

It is worth also noting that regularity of solution in Morrey spaces have been recently derived in [26] for linear, non divergence form operators with oblique derivative boundary condition by means of estimates for singular integrals of CalderónZygmund type. Moreover, the Morrey regularity results from Section 5 could be extended in the more general framework of generalized Morrey spaces (see [16, 25, 27]).

Throughout the paper, the letter $c$ will denote a universal constant that can be explicitly computed in terms of known quantities such as $n, L, \nu, p, \omega$ and the geometric structure on $Q$. The exact value of $c$ may vary from one occurrence to another.

Acknowledgements. S. Byun was supported by Basic Science Research Program through the National Research Foundation of Korea (NRF) grant funded by the Korea government (MEST) (NRF-2014K2A2A2000796). The work of D. K. Palagachev and L. G. Softova is part of the INdAM-GNAMPA Project 2015 "Regolarità delle soluzioni di problemi al bordo per operatori differenziali su domini non regolari o non limitati".

\section{Weighted Lebesgue spaces in parabolic settings}

Our aim is to establish a global weighted estimate of Calderón-Zygmund type for the weak solution of (1.1) and let us start with describing the properties of the class of weights considered. For this goal, we will use the parabolic metric given in Stein [28] by the function

$$
\rho(x, t)=\sqrt{\frac{|x|^{2}+\sqrt{|x|^{4}+4|t|^{2}}}{2}} \quad(x, t) \in \mathbf{R}^{n+1} .
$$

It is proved by Fabes and Rivière in [14] that $\rho$ defines a metric in $\mathbf{R}^{n+1}$ and the "balls" with respect to it, centered at $(x, t)$ and of radius $r>0$, are the ellipsoids $\mathcal{E}=\mathcal{E}_{r}(x, t)$

$$
\mathcal{E}_{r}(x, t)=\left\{(y, \tau) \in \mathbf{R}^{n+1}: \frac{|x-y|^{2}}{r^{2}}+\frac{|t-\tau|^{2}}{r^{4}}<1\right\}
$$

or, in an alternative way,

$$
\mathcal{E}_{r}(x, t)=\left\{(y, \tau) \in \mathbf{R}^{n+1}: \rho(x-y, t-\tau)<r\right\} .
$$

Let $\mu$ be a non-negative Borel measure on $\mathbf{R}^{n+1}$ with the property $\mu\left(\mathbf{R}^{n+1}\right)>0$. In the particular case when $\mu$ is the Lebesgue measure, then $\mu\left(\mathcal{E}_{r}\right)=\left|\mathcal{E}_{r}\right|=c r^{n+2}$ with a positive constant $c=c(n)$. Let us note that for all points $(x, t),(y, \tau) \in \mathbf{R}^{n+1}$ and $r>0$, the collection of such ellipsoids and the measure that we postulate satisfy the following properties (cf. [28]): There exist constants $c_{1}$ and $c_{2}$, both greater than 1 and depending on $n$, such that

(i) $\mathcal{E}_{r}(x, t) \cap \mathcal{E}_{r}(y, \tau) \neq \emptyset$ implies $\mathcal{E}_{r}(y, \tau) \subset \mathcal{E}_{c_{1} r}(x, t)$;

(ii) $\mu\left(\mathcal{E}_{c_{1} r}(x, t)\right) \leq c_{2} \mu\left(\mathcal{E}_{r}(x, t)\right)$;

(iii) For each open set $U$ and $r>0$, the function $(x, t) \rightarrow \mu\left(\mathcal{E}_{r}(x, t) \cap U\right)$ is continuous. 
Statement (i) guarantees the engulfing property crucial in the Vitali-type covering lemma that we are going to use, while the doubling type property (ii) of the measure just allows one to exploit the first statement. In our further considerations we shall also use the collection of cylinders $\mathcal{C} \equiv \mathcal{C}_{r}(x, t)=\mathcal{C}_{r}\left(x_{1}, x^{\prime}, t\right)$ defined as

$$
\mathcal{C}_{r}(x, t)=\left\{\left(y_{1}, y^{\prime}, \tau\right) \in \mathbf{R}^{n+1}:\left|x_{1}-y_{1}\right|<r, \rho\left(x^{\prime}-y^{\prime}, t-\tau\right)<r\right\},
$$

or, in an alternate way

$$
\mathcal{C}_{r}(x, t)=\left\{\left(y_{1}, y^{\prime}, \tau\right) \in \mathbf{R}^{n+1}:\left|x_{1}-y_{1}\right|<r, \max \left\{\left|x^{\prime}-y^{\prime}\right|, \sqrt{|t-\tau|}\right\}<r\right\}
$$

with the Lebesgue measure $\left|\mathcal{C}_{r}\right|$ comparable to $r^{n+2}$, and where we have set $x^{\prime}=$ $\left(x_{2}, \ldots, x_{n}\right)$.

Remark 2.1. It is not difficult to verify that (i), (ii) and (iii) hold for the collections (2.1) and (2.2). In what follows we will use the equivalence of these structures without explicit references (cf. [28]). All definitions given over ellipsoids $\mathcal{E}_{r}$ hold also over the cylinders $\mathcal{C}_{r}$.

In case of $\mathbf{R}^{n}$ we shall use also the following collection of cylinders

$$
\mathcal{C}_{r}^{\prime}(x)=\left\{y \in \mathbf{R}^{n}:\left|x_{1}-y_{1}\right|<r,\left|x^{\prime}-y^{\prime}\right|<r\right\} .
$$

To define the functional spaces to be used in the sequel, we need to recall the definition and some properties of the Muckenhoupt weights (cf. García-Cuerva and Rubio de Francia [15], Stein [28] and Torchinsky [29]). Let $\mathcal{M}$ denote the Hardy-Littlewood maximal operator on $\mathbf{R}^{n+1}$

$$
\mathcal{M} f(x, t)=\sup _{r>0} \frac{1}{\left|\mathcal{E}_{r}(x, t)\right|} \int_{\mathcal{E}_{r}(x, t)}|f(y, \tau)| d y d \tau, \quad f \in L_{\mathrm{loc}}^{1}\left(\mathbf{R}^{n+1}\right) .
$$

If $D$ is a bounded domain in $\mathbf{R}^{n+1}$ and $f \in L^{1}(D)$, then $\mathcal{M} f=\mathcal{M} \bar{f}$, where $\bar{f}$ is the zero extension of $f$ in the whole space. It is well known that $\mathcal{M}$ is bounded sub-linear operator from $L^{q}$ to itself for all $q>1$. That is, if $f \in L^{q}\left(\mathbf{R}^{n+1}\right), q \in(1, \infty)$, then

$$
\int_{\mathbf{R}^{n+1}}|\mathcal{M} f(x, t)|^{q} d \mu(x, t) \leq c \int_{\mathbf{R}^{n+1}}|f(x, t)|^{q} d \mu(x, t)
$$

for some positive constant $c=c(q, n)$, where $d \mu=d x d t$ is the Lebesgue measure. It turns out that the estimate (2.3) still holds true when $d \mu=\omega(x, t) d x d t$, where $\omega: \mathbf{R}^{n+1} \rightarrow \mathbf{R}_{+}$is a positive, locally integrable function, satisfying the following $A_{q}$-condition

$$
\left(\frac{1}{|\mathcal{E}|} \int_{\mathcal{E}} \omega(x, t) d x d t\right)\left(\frac{1}{|\mathcal{E}|} \int_{\mathcal{E}} \omega(x, t)^{-\frac{1}{q-1}} d x d t\right)^{q-1} \leq A<\infty
$$

for all $\mathcal{E}$ in $\mathbf{R}^{n+1}$. It is proved in [22] that (2.4) is a necessary and sufficient condition in order (2.3) to hold. By this reason, $\omega$ is commonly called Muckenhoupt weight lying in the class $A_{q}$, and the smallest constant $A$ for which (2.4) holds is denoted by $[\omega]_{q}$. If $q=1$, we say that $\omega \in A_{1}$ when

$$
\frac{1}{|\mathcal{E}|} \int_{\mathcal{E}} \omega(x, t) d x d t \leq A \underset{\mathcal{E}}{\operatorname{essinf}} \omega(x, t)
$$

There is an alternative way of defining $A_{q}$, closely related to the maximal inequality (2.3). For any non-negative, locally integrable function $f$ and any ellipsoid $\mathcal{E}$, the weight $\omega$ belongs to $A_{q}, 1 \leq q<\infty$, if and only if

$$
\left(\frac{1}{|\mathcal{E}|} \int_{\mathcal{E}} f(x, t) d x d t\right)^{q} \leq \frac{A}{\omega(\mathcal{E})} \int_{\mathcal{E}} f^{q}(x, t) \omega(x, t) d x d t<\infty
$$


for some $A=A(q, n)>0$, where

$$
\omega(\mathcal{E})=\int_{\mathcal{E}} \omega(x, t) d x d t<\infty
$$

is the measure of $\mathcal{E}$ with respect to $d \mu=\omega(x, t) d x d t$. The smallest $A$ for which (2.6) is valid equals $[\omega]_{q}$. It is an immediate consequence of (2.6) that whenever $\omega \in A_{q}$ then it satisfies the doubling property, that is,

$$
\omega\left(\mathcal{E}_{2 r}\right) \leq c(n, q) \omega\left(\mathcal{E}_{r}\right)
$$

Actually, apply (2.6) with $\mathcal{E}=\mathcal{E}_{2 r}$ and $f=\chi_{\mathcal{E}_{r}}$ that gives $(2.8)$ with $c=[\omega]_{q} 2^{q(n+2)}$. The doubling property of $\omega$, together with (2.6), shows that in the definition (2.4) we could have replaced the family of ellipsoids $\left\{\mathcal{E}_{r}\right\}_{r>0}$ by a family of cylinders $\left\{\mathcal{C}_{r}\right\}_{r>0}$ or other such equivalent families, as it is noted in Remark 2.1.

A noteworthy feature of the $A_{q}$ classes is that these increase with $q$, that is, if $\omega \in A_{q}$, then $\omega \in A_{p}$ whenever $p \geq q$ and $[\omega]_{p} \leq[\omega]_{q}$.

Another important characteristic of the Muckenhoupt weights is the strong doubling property (see [29, Theorem IX.2.1] or [21, Lemma 3.3]). Moreover, as proved in [28, Section V.5.3], for each weight $\omega \in A_{q}, q>1$, there exist $\omega_{1}$ and $\omega_{2}$ in $A_{1}$ so that $\omega=\omega_{1} \omega_{2}^{1-q}$. This, along with [29, Proposition IX.4.5], gives that $\omega$ satisfies the reverse Hölder inequality and reverse doubling property. Unifying the both doubling conditions, one can observe that for each $\mathcal{E}$ and each measurable subset $A \subset \mathcal{E}$, there exist positive constants $c_{1}$ and $\tau_{1} \in(0,1)$ such that

$$
\frac{1}{[\omega]_{q}}\left(\frac{|A|}{|\mathcal{E}|}\right)^{q} \leq \frac{\omega(A)}{\omega(\mathcal{E})} \leq c_{1}\left(\frac{|A|}{|\mathcal{E}|}\right)^{\tau_{1}}
$$

where $c_{1}$ and $\tau_{1}$ depend on $[\omega]_{q}, n$ and $q$, but are independent of $\mathcal{E}$ and $A$. Let us note that the lower bound in (2.9) is the above mentioned strong doubling property, while the upper one is the reverse doubling property.

Given a measurable and non-negative weight $\omega(x, t)$, the weighted Lebesgue space $L_{\omega}^{q}\left(\mathbf{R}^{n+1}\right), q>1$, is the collection of all measurable functions $f$ for which

$$
\|f\|_{L_{\omega}^{q}\left(\mathbf{R}^{n+1}\right)}^{q}=\int_{\mathbf{R}^{n+1}}|f(x, t)|^{q} \omega(x, t) d x d t<\infty .
$$

As already mentioned above, the famous result of Muckenhoupt [22] states that $\omega \in A_{q}$ is a necessary and sufficient condition ensuring that the Hardy-Littlewood maximal operator maps $L_{\omega}^{q}$ into itself (see also [29, Theorem IX.4.1]). Summarizing, we have

Lemma 2.2. Suppose $\omega(x, t) \in A_{q}, q \in(1, \infty)$. Then there exists a positive constant $c=c(q, n)$ such that

$$
\frac{1}{c}\|f\|_{L_{\omega}^{q}\left(\mathbf{R}^{n+1}\right)} \leq\|\mathcal{M} f\|_{L_{\omega}^{q}\left(\mathbf{R}^{n+1}\right)} \leq c\|f\|_{L_{\omega}^{q}\left(\mathbf{R}^{n+1}\right)}
$$

whenever $f \in L_{\omega}^{q}\left(\mathbf{R}^{n+1}\right)$.

Example 2.3. The weight function $\omega(x, t)=\rho(x, t)^{\alpha}$ belongs to $A_{q}$ with $q \in$ $(1, \infty)$ if and only if $-(n+2)<\alpha<(q-1)(n+2)$. 


\section{Assumptions and main result}

For each cylinder $\mathcal{C}_{r}(y, \tau)=\mathcal{C}_{r}\left(y_{1}, y^{\prime}, \tau\right)$ and for a fixed $x_{1} \in\left(y_{1}-r, y_{1}+r\right)$ we set $\mathcal{C}_{r}^{x_{1}}(y, \tau)$ to denote the $x_{1}$-slice of $\mathcal{C}_{r}(y, \tau)$, that is,

$$
\mathcal{C}_{r}^{x_{1}}(y, \tau)=\left\{\left(x^{\prime}, t\right) \in \mathbf{R}^{n-1} \times \mathbf{R}:(x, t)=\left(x_{1}, x^{\prime}, t\right) \in \mathcal{C}_{r}(y, \tau)\right\} .
$$

Then we define the integral average

$$
\overline{\mathbf{a}}_{\mathcal{C}_{r}^{x_{1}}(y, \tau)}\left(x_{1}\right)=\frac{1}{\left|\mathcal{C}_{r}^{x_{1}}(y, \tau)\right|} \int_{\mathcal{C}_{r}^{x_{1}}(y, \tau)} \mathbf{a}\left(x_{1}, x^{\prime}, t\right) d x^{\prime} d t
$$

Definition 3.1. We say that the couple $(\mathbf{a}, \Omega)$ is $(\delta, R)$-vanishing of co-dimension 1, if the following properties are satisfied:

- For every point $(y, \tau) \in Q$ and for every number $r \in\left(0, \frac{1}{3} R\right]$ with

$$
\operatorname{dist}(y, \partial \Omega)>\sqrt{2} r
$$

there exists a coordinate system depending on $(y, \tau)$ and $r$, whose variables we still denote by $(x, t)$ so that in this new coordinate system $(y, \tau)$ is the origin and

$$
\frac{1}{\left|\mathcal{C}_{r}(0,0)\right|} \int_{\mathcal{C}_{r}(0,0)}\left|\mathbf{a}(x, t)-\overline{\mathbf{a}}_{\mathcal{C}_{r}^{x_{1}}(0,0)}\left(x_{1}\right)\right|^{2} d x d t \leq \delta^{2}
$$

- For any point $(y, \tau) \in Q$ and for every number $r \in\left(0, \frac{1}{3} R\right]$ such that

$$
\operatorname{dist}(y, \partial \Omega)=\operatorname{dist}\left(y, x_{0}\right) \leq \sqrt{2} r
$$

for some $x_{0} \in \partial \Omega$, there exists a coordinate system depending on $(y, \tau)$ and $r$, whose variables we still denote by $(x, t)$ such that in this new coordinate system $\left(x_{0}, \tau\right)$ is the origin,

$$
\begin{aligned}
\Omega \cap\left\{x \in \mathcal{C}_{3 r}^{\prime}(0): x_{1}>3 r \delta\right\} & \subset \Omega \cap \mathcal{C}_{3 r}^{\prime}(0) \\
& \subset \Omega \cap\left\{x \in \mathcal{C}_{3 r}^{\prime}(0): x_{1}>-3 r \delta\right\}
\end{aligned}
$$

and

$$
\frac{1}{\left|\mathcal{C}_{3 r}(0,0)\right|} \int_{\mathcal{C}_{3 r}(0,0)}\left|\mathbf{a}(x, t)-\overline{\mathbf{a}}_{\mathcal{C}_{3 r}^{x_{1}}(0,0)}\left(x_{1}\right)\right|^{2} d x d t \leq \delta^{2}
$$

We add some comments regarding the above definition. Thanks to the scaling invariance property, one can take for simplicity $R=1$ or any other constant bigger than 1 . On the other hand $\delta$ is a small positive constant, being invariant under such a scaling argument. If $\mathbf{a}$ is $(\delta, R)$-vanishing of co-dimension 1 , then for each point and for each sufficiently small scale, there is a coordinate system so that the coefficients have small oscillation in $\left(x^{\prime}, t\right)$-variables while these are only measurable in the $x_{1}$ variable and therefore may have arbitrary jumps with respect to it. In addition, the boundary of the domain is $(\delta, R)$-Reifenberg flat (see [23]) and the coefficients have a small oscillation along the flat direction $x^{\prime}$ of the boundary and are only measurable along the normal direction $x_{1}$. The number $\sqrt{2} r$ in (3.1) is selected for convenience. It comes from the reason that we need to take an enough size of the cylinders in (3.2) so that there is a room to have the rotation of $\mathcal{C}_{r}(y, \tau)$ in any spatial direction.

We suppose that the right-hand side of the equation in (1.1) belongs to some weighted Lebesgue space, precisely

which implies

$$
|\mathbf{F}|^{2} \in L_{\omega}^{\frac{p}{2}}(Q), \quad \omega \in A_{\frac{p}{2}}, \quad p \in(2, \infty),
$$

$$
\mathbf{F} \in L_{\omega}^{p}(Q), \quad \omega \in A_{\frac{p}{2}} \subset A_{p}, \quad p \in(2, \infty)
$$


Then we get from Hölder's inequality, (2.4), (2.7) and (2.10) that

$$
\begin{aligned}
\|\mathbf{F}\|_{L^{2}(Q)}^{2} & =\int_{Q}|\mathbf{F}(x, t)|^{2} \omega^{\frac{2}{p}}(x, t) \omega^{-\frac{2}{p}}(x, t) d x d t \\
& \leq\left(\int_{Q}\left(|\mathbf{F}(x, t)|^{2}\right)^{\frac{p}{2}} \omega(x, t) d x d t\right)^{\frac{2}{p}}\left(\int_{Q} \omega(x, t)^{-\frac{2}{p-2}} d x d t\right)^{\frac{p-2}{p}} \\
& =\left\||\mathbf{F}|^{2}\right\|_{L_{\omega}^{\frac{p}{2}(Q)}}|Q|^{\frac{p-2}{p}}\left(\frac{1}{|Q|} \int_{Q} \omega(x, t)^{-\frac{2}{p-2}} d x d t\right)^{\frac{p-2}{p}} \\
& \leq\left\||\mathbf{F}|^{2}\right\|_{L_{\omega}^{\frac{p}{2}(Q)}}|Q| \omega(Q)^{-\frac{2}{p}}[\omega]_{\frac{p}{2}}^{\frac{2}{p}} .
\end{aligned}
$$

Hence we have

$$
\frac{1}{c}\|\mathbf{F}\|_{L^{2}(Q)}^{2} \leq\left\||\mathbf{F}|^{2}\right\|_{L_{w}^{\frac{p}{2}(Q)}}<\infty
$$

with a constant $c=|Q| \omega(Q)^{-\frac{2}{p}}[\omega]_{\frac{p}{2}}^{\frac{2}{p}}$, which ensures the existence of a unique weak solution $u$ of the equation (1.1) (cf. $[2,6]$ ).

We now state the main result of the paper.

Theorem 3.2. Let $p \in(2, \infty), \omega \in A_{\frac{p}{2}}$ and assume (1.2). Then there exists a small positive constant $\delta=\delta(n, L, \nu, p, \omega, Q)$ such that if the couple $(\mathbf{a}, \Omega)$ is $(\delta, R)$ vanishing of co-dimension 1 and $\mathbf{F} \in L_{\omega}^{p}(Q)$, then the spatial gradient $D u$ of the weak solution $u$ of (1.1) belongs to $L_{\omega}^{p}(Q)$ and the following estimate holds

$$
\|D u\|_{L_{\omega}^{p}(Q)} \leq c\|\mathbf{F}\|_{L_{\omega}^{p}(Q)}
$$

with a constant $c$ depending on $n, L, \nu, p, \omega$ and $Q$.

The present work is a natural extension of the previous paper [5] which deals with the regularity problem for parabolic equations in classical (unweighted, $\omega(x, t) \equiv 1$ ) Lebesgue classes.

Here with a natural parabolic Muckenhoupt weight for the problem (1.1), we first find a correct version for the weight of the Vitali covering lemma, and verify the hypotheses of this covering lemma from the perturbation results for the unweighted case and comparable relationships between the Lebesgue and the weighted measures. We then apply the covering lemma to derive a weighted power decay estimate of the upper level sets for the Hardy-Littlewood maximal function of the spatial gradient of the weak solution. The required estimate in the main result follows then by the standard procedure of summation over the level sets.

\section{Gradient estimates in $L_{\omega}^{p}$}

Because of the scaling invariance property of the Reifenberg flat domains (cf. [5, Lemma 5.2] for instance), we can take $R=1$ hereafter. The next result is a parabolic counterpart of an elliptic weighted covering lemma obtained in [21, Lemma 3.8].

Lemma 4.1. Suppose $\Omega$ is a bounded $(\delta, 1)$-Reifenberg flat domain (that is, (3.3) is verified) and $\omega(x, t) \in A_{q}, q \in(1, \infty)$. Let $\mathfrak{C} \subset \mathfrak{D} \subset Q$ be measurable subsets of $Q$ satisfying the following conditions:

- there exists $\varepsilon \in(0,1)$ such that for each $(y, \tau) \in Q$

$$
\omega\left(\mathfrak{C} \cap \mathcal{C}_{1}(y, \tau)\right)<\epsilon \omega\left(\mathcal{C}_{1}(y, \tau)\right) ;
$$


- for each $(y, \tau) \in Q$ and $r>0$

$$
\omega\left(\mathfrak{C} \cap \mathcal{C}_{r}(y, \tau)\right) \geq \varepsilon \omega\left(\mathcal{C}_{r}(y, \tau)\right) \quad \text { implies } \quad Q \cap \mathcal{C}_{r}(y, \tau) \subset \mathfrak{D} .
$$

Then

$$
\omega(\mathfrak{C}) \leq \varepsilon[\omega]_{q}^{2}\left(\frac{10 \sqrt{2}}{1-\delta}\right)^{q(n+2)} \omega(\mathfrak{D})
$$

Proof. Fix $(y, \tau) \in Q$ and for each $r>0$ define the function

$$
\Theta(r)=\frac{\omega\left(\mathfrak{C} \cap \mathcal{C}_{r}(y, \tau)\right)}{\omega\left(\mathcal{C}_{r}(y, \tau)\right)} .
$$

We have $\Theta \in C^{0}(0, \infty), \Theta(1)<\varepsilon$ by (4.1), and $\Theta(0)=\lim _{r \rightarrow 0_{+}} \Theta(r)=1$ according to the Lebesgue Differentiation Theorem. Therefore, for almost all $(y, \tau) \in \mathfrak{C}$, there exists $r_{(y, \tau)} \in(0,1)$ such that $\Theta\left(r_{(y, \tau)}\right)=\varepsilon$ and $\Theta(r)<\varepsilon$ for all $r>r_{(y, \tau)}$.

Define the family of cylinders $\left\{\mathcal{C}_{r_{(y, \tau)}}(y, \tau)\right\}_{(y, \tau) \in \mathfrak{C}}$ which forms an open covering of $\mathfrak{C}$. By the Vitali lemma (cf. [28, Lemma I.3.1]), there exists a disjoint sub-collection $\left\{\mathcal{C}_{r_{i}}\left(y_{i}, \tau_{i}\right)\right\}_{i \geq 1}$ with $r_{i}=r_{\left(y_{i}, \tau_{i}\right)} \in(0,1),\left(y_{i}, \tau_{i}\right) \in \mathfrak{C}$ such that $\Theta\left(r_{i}\right)=\varepsilon$ and

$$
\sum_{i \geq 1}\left|\mathcal{C}_{r_{i}}\left(y_{i}, \tau_{i}\right)\right| \geq c|\mathfrak{C}|, \quad \mathfrak{C} \subset \bigcup_{i \geq 1} \mathcal{C}_{5 r_{i}}\left(y_{i}, \tau_{i}\right), \quad \mathfrak{C}=\bigcup_{i \geq 1}\left(\mathfrak{C} \cap \mathcal{C}_{5 r_{i}}\left(y_{i}, \tau_{i}\right)\right)
$$

for some $c=c(n)>0$.

Since $\Theta\left(5 r_{i}\right)<\varepsilon$, we have by $(2.9)$

$$
\begin{aligned}
\omega\left(\mathfrak{C} \cap \mathcal{C}_{5 r_{i}}\left(y_{i}, \tau_{i}\right)\right) & <\varepsilon \omega\left(\mathcal{C}_{5 r_{i}}\left(y_{i}, \tau_{i}\right)\right) \leq \varepsilon[\omega]_{q}\left(\frac{\left|\mathcal{C}_{5 r_{i}}\left(y_{i}, \tau_{i}\right)\right|}{\left|\mathcal{C}_{r_{i}}\left(y_{i}, \tau_{i}\right)\right|}\right)^{q} \omega\left(\mathcal{C}_{r_{i}}\left(y_{i}, \tau_{i}\right)\right) \\
& =\varepsilon[\omega]_{q} 5^{q(n+2)} \omega\left(\mathcal{C}_{r_{i}}\left(y_{i}, \tau_{i}\right)\right) .
\end{aligned}
$$

In order to employ (4.2), we have to estimate the ratio $\frac{\omega\left(\mathcal{C}_{r_{i}}\left(y_{i}, \tau_{i}\right)\right)}{\omega\left(Q \cap \mathcal{C}_{r_{i}}\left(y_{i}, t_{i}\right)\right)}$. For this goal, making use of the bound

$$
\sup _{0<r<1} \sup _{(y, \tau) \in Q} \frac{\left|\mathcal{C}_{r}(y, \tau)\right|}{\left|Q \cap \mathcal{C}_{r}(y, \tau)\right|} \leq\left(\frac{2 \sqrt{2}}{1-\delta}\right)^{n+2},
$$

obtained in [5, 7], and the doubling condition (2.9), we get

$$
\omega\left(\mathcal{C}_{r_{i}}\left(y_{i}, \tau_{i}\right)\right) \leq[\omega]_{q}\left(\frac{2 \sqrt{2}}{1-\delta}\right)^{q(n+2)} \omega\left(Q \cap \mathcal{C}_{r_{i}}\left(y_{i}, \tau_{i}\right)\right) .
$$

Now we have

$$
\begin{aligned}
\omega(\mathfrak{C}) & \leq \omega\left(\bigcup_{i \geq 1}\left(\mathfrak{C} \cap \mathcal{C}_{5 r_{i}}\left(y_{i}, \tau_{i}\right)\right)\right) \leq \sum_{i \geq 1} \omega\left(\mathfrak{C} \cap \mathcal{C}_{5 r_{i}}\left(y_{i}, \tau_{i}\right)\right) \\
& <\varepsilon \sum_{i \geq 1} \omega\left(\mathcal{C}_{5 r_{i}}\left(y_{i}, \tau_{i}\right)\right) \leq \varepsilon[\omega]_{q} 5^{q(n+2)} \sum_{i \geq 1} \omega\left(\mathcal{C}_{r_{i}}\left(y_{i}, \tau_{i}\right)\right) \\
& \leq \varepsilon[\omega]_{q}^{2}\left(\frac{10 \sqrt{2}}{1-\delta}\right)^{q(n+2)} \sum_{i \geq 1} \omega\left(Q \cap \mathcal{C}_{r_{i}}\left(y_{i}, \tau_{i}\right)\right) .
\end{aligned}
$$


Having in mind that $\left\{\mathcal{C}_{r_{i}}\left(y_{i}, \tau_{i}\right)\right\}$ are mutually disjoint, $\Theta\left(r_{i}\right)=\varepsilon$ and (4.2), we get

$$
\omega(\mathfrak{C}) \leq \varepsilon[\omega]_{q}^{2}\left(\frac{10 \sqrt{2}}{1-\delta}\right)^{q(n+2)} \omega\left(\bigcup_{i \geq 1} Q \cap \mathcal{C}_{r_{i}}\left(y_{i}, \tau_{i}\right)\right) \leq \varepsilon[\omega]_{q}^{2}\left(\frac{10 \sqrt{2}}{1-\delta}\right)^{q(n+2)} \omega(\mathfrak{D})
$$

In the following we recall an approximation lemma obtained for the unweighted spaces in [12, Corollary 8.4], [5, Lemma 5.3] and [7, Lemma 5.5].

Lemma 4.2. Assume (1.2) and let $u$ be a weak solution of (1.1). Then there is a constant $\lambda_{1}=\lambda_{1}(\nu, n)>1$ such that for each $\varepsilon \in(0,1)$ there exists $\delta=\delta(\varepsilon)>0$ such that if $\mathbf{a}$ is $(\delta, 1)$-vanishing of co-dimension 1 and if $\mathcal{C}_{r}(y, \tau)$ satisfies

$$
\left|\left\{(x, t) \in Q: \mathcal{M}\left(|D u|^{2}\right)>\lambda_{1}^{2}\right\} \cap \mathcal{C}_{r}(y, \tau)\right| \geq \varepsilon\left|\mathcal{C}_{r}(y, \tau)\right|,
$$

then we have

$$
Q \cap \mathcal{C}_{r}(y, \tau) \subset\left\{(x, t) \in Q: \mathcal{M}\left(|D u|^{2}\right)>1\right\} \cup\left\{\mathcal{M}\left(|\mathbf{F}|^{2}\right)>\delta^{2}\right\}
$$

We need now to establish a weighted version of the lemma cited above. For this goal, for any weak solution $u$ of (1.1) we set

$$
\mathfrak{C}=\left\{(x, t) \in Q: \mathcal{M}\left(|D u|^{2}\right)>\lambda_{1}^{2}\right\}
$$

and

$$
\mathfrak{D}=\left\{(x, t) \in Q: \mathcal{M}\left(|D u|^{2}\right)>1\right\} \cup\left\{\mathcal{M}\left(|\mathbf{F}|^{2}\right)>\delta^{2}\right\}
$$

with $\lambda_{1}$ and $\delta$ as in Lemma 4.2. The next assertion shows that the assumption (4.2) holds for the such defined sets $\mathfrak{C}$ and $\mathfrak{D}$.

Lemma 4.3. Let $\omega \in A_{q}, q \in(1, \infty)$. Assume that $\mathbf{a}$ is $(\delta, 1)$-vanishing of co-dimension 1 and for each $r>0$ and almost all $(y, \tau) \in Q, \mathcal{C}_{r}(y, \tau)$ satisfies

$$
\Theta(r)=\frac{\omega\left(\mathfrak{C} \cap \mathcal{C}_{r}(y, \tau)\right)}{\omega\left(\mathcal{C}_{r}(y, \tau)\right)} \geq \varepsilon
$$

Then we have $Q \cap \mathcal{C}_{r}(y, \tau) \subset \mathfrak{D}$.

Proof. The reverse doubling property of $\omega$ (the upper bound in (2.9)) gives that

$$
\varepsilon \leq \frac{\omega\left(\mathfrak{C} \cap \mathcal{C}_{r}(y, \tau)\right)}{\omega\left(\mathcal{C}_{r}(y, \tau)\right)} \leq c_{1}\left(\frac{\left|\mathfrak{C} \cap \mathcal{C}_{r}(y, \tau)\right|}{\left|\mathcal{C}_{r}(y, \tau)\right|}\right)^{\tau_{1}}
$$

Hence

$$
\left|\mathfrak{C} \cap \mathcal{C}_{r}(y, \tau)\right| \geq\left(\frac{\varepsilon}{c_{1}}\right)^{\frac{1}{\tau_{1}}}\left|\mathcal{C}_{r}(y, \tau)\right|
$$

The assertion holds after applying Lemma 4.2 with $\varepsilon$ replaced by $\left(\frac{\varepsilon}{c_{1}}\right)^{\frac{1}{\tau_{1}}}$.

We are going to derive now the power decay estimate of the upper level set $\mathfrak{C}$ with respect to $A_{\frac{p}{2}}$-weights.

Lemma 4.4. Under the assumptions of Lemma 4.3 we suppose additionally that

$$
\Theta(1)=\frac{\omega\left(\mathfrak{C} \cap \mathcal{C}_{1}(y, \tau)\right)}{\omega\left(\mathcal{C}_{1}(y, \tau)\right)}<\varepsilon
$$


with $\mathfrak{C}$ as in (4.4). Then for each $k=1,2, \ldots$, we have

$$
\begin{aligned}
\omega\left(\left\{(x, t) \in Q: \mathcal{M}\left(|D u|^{2}\right)>\right.\right. & \left.\left.\lambda_{1}^{2 k}\right\}\right) \leq \varepsilon_{1}^{k} \omega\left(\left\{(x, t) \in Q: \mathcal{M}\left(|D u|^{2}\right)>1\right\}\right) \\
& +\sum_{i=1}^{k} \varepsilon_{1}^{i} \omega\left(\left\{(x, t) \in Q: \mathcal{M}\left(|\mathbf{F}|^{2}\right)>\delta^{2} \lambda_{1}^{2(k-i)}\right\}\right)
\end{aligned}
$$

where $\varepsilon_{1}=\varepsilon[\omega]_{\frac{p}{2}}^{2}\left(\frac{10 \sqrt{2}}{1-\delta}\right)^{\frac{p}{2}(n+2)}$.

Proof. Lemma 4.3 and condition (4.6) ensure the validity of the hypotheses of Lemma 4.1 for the sets (4.4) and (4.5). Thus, we get by (4.3)

$$
\begin{aligned}
\omega\left(\left\{(x, t) \in Q: \mathcal{M}\left(|D u|^{2}\right)>\lambda_{1}^{2}\right\}\right) \leq & \varepsilon_{1} \omega\left(\left\{(x, t) \in Q: \mathcal{M}\left(|D u|^{2}\right)>1\right\}\right) \\
& +\varepsilon_{1} \omega\left(\left\{(x, t) \in Q: \mathcal{M}\left(|\mathbf{F}|^{2}\right)>\delta^{2}\right\}\right),
\end{aligned}
$$

where $\varepsilon_{1}=\varepsilon[\omega]_{\frac{p}{2}}\left(\frac{10 \sqrt{2}}{1-\delta}\right)^{\frac{p}{2}(n+2)}$.

The last inequality is exactly (4.7) with $k=1$. Further, we proceed with the proof by induction, as it is done in [1, Corollary 4.15]. Suppose that (4.7) holds true for each weak solution of (1.1) and for some $k>1$. Define the functions $u_{1}=\frac{u}{\lambda_{1}}$ and $\mathbf{F}_{1}=\frac{\mathbf{F}}{\lambda_{1}}$. It is easy to see that $u_{1}$ is a weak solution to the problem (1.1) with a righthand side $\mathbf{F}_{1}$. Hence, (4.6) and Lemma 4.3 hold with the sets $\mathfrak{C}$ and $\mathfrak{D}$ corresponding to $u_{1}$ as defined by (4.4) and (4.5) and according to (4.7), the inductive assumption holds true for $u_{1}$ with the same $k>1$. The definition of $u_{1}$ ensures the inductive passage from $k$ to $k+1$ for $u$. Namely,

$$
\begin{aligned}
\omega & \left(\left\{(x, t) \in Q: \mathcal{M}\left(|D u|^{2}\right)>\lambda_{1}^{2(k+1)}\right\}\right)=\omega\left(\left\{(x, t) \in Q: \mathcal{M}\left(\left|D u_{1}\right|^{2}\right)>\lambda_{1}^{2 k}\right\}\right) \\
\leq & \varepsilon_{1}^{k} \omega\left(\left\{(x, t) \in Q: \mathcal{M}\left(\left|D u_{1}\right|^{2}\right)>1\right\}\right) \\
& +\sum_{i=1}^{k} \varepsilon_{1}^{i} \omega\left(\left\{(x, t) \in Q: \mathcal{M}\left(\left|\mathbf{F}_{1}\right|^{2}\right)>\delta^{2} \lambda_{1}^{2(k-i)}\right\}\right) \\
= & \varepsilon_{1}^{k} \omega\left(\left\{(x, t) \in Q: \mathcal{M}\left(|D u|^{2}\right)>\lambda_{1}^{2}\right\}\right) \\
& +\sum_{i=1}^{k} \varepsilon_{1}^{i} \omega\left(\left\{(x, t) \in Q: \mathcal{M}\left(|\mathbf{F}|^{2}\right)>\delta^{2} \lambda_{1}^{2(k-i)} \lambda^{2}\right\}\right) \\
\leq & \varepsilon_{1}^{k+1} \omega\left(\left\{(x, t) \in Q: \mathcal{M}\left(|D u|^{2}\right)>1\right\}\right) \\
& +\sum_{i=1}^{k+1} \varepsilon_{1}^{i} \omega\left(\left\{(x, t) \in Q: \mathcal{M}\left(|\mathbf{F}|^{2}\right)>\delta^{2} \lambda_{1}^{2(k+1-i)}\right\}\right) .
\end{aligned}
$$

The next result follows directly from [21, Lemma 3.7].

Lemma 4.5. Let $h \in L^{1}(Q)$ be a non-negative function, $\omega$ be an $A_{q}$-weight, $q \in(1, \infty)$ and $\theta>0, \Lambda>1$ be constants. Then $h \in L_{\omega}^{q}(Q)$ if and only if

$$
\mathcal{S}:=\sum_{k \geq 1} \Lambda^{k q} \omega\left(\left\{(x, t) \in Q: h(x, t)>\theta \Lambda^{k}\right\}\right)<\infty .
$$

Moreover,

$$
c^{-1} \mathcal{S} \leq\|h\|_{L_{\omega}^{q}(Q)}^{q} \leq c(\omega(Q)+\mathcal{S})
$$

where $c=c(\theta, \Lambda, q)$. 
We are in a position now to prove Theorem 3.2.

Proof of Theorem 3.2. Since $\omega \in A_{\frac{p}{2}}$ then $\omega \in A_{p}$ with $[\omega]_{p} \leq[\omega]_{\frac{p}{2}}$. Recall that $\mathbf{F} \in L_{\omega}^{p}(Q)$ and from the scaling invariance property of (1.1) under a normalization, we assume that $\|\mathbf{F}\|_{L_{\omega}^{p}(Q)} \leq \delta$ with $\delta>0$ small enough. Hence

$$
\|\mathbf{F}\|_{L_{\omega}^{p}(Q)}^{2}=\left\||\mathbf{F}|^{2}\right\|_{L_{\omega}^{\frac{p}{2}(Q)}} \leq \delta^{2} .
$$

Then we need to prove boundedness of the norm of the gradient $\|D u\|_{L_{\omega}^{p}(Q)}$. Because of the properties of the maximal function (see Lemma 2.2), it is enough to get

$$
\left\|\mathcal{M}\left(|D u|^{2}\right)\right\|_{L_{\omega}^{\frac{p}{2}(Q)}} \leq c \text {. }
$$

For this goal, we apply Lemma 4.5 with $h=\mathcal{M}\left(|D u|^{2}\right), \Lambda=\lambda_{1}^{2}, q=\frac{p}{2}, \theta=1$. By the reverse doubling property (2.9), we have

$$
\frac{\omega\left(\mathfrak{C} \cap \mathcal{C}_{1}(y, \tau)\right)}{\omega\left(\mathcal{C}_{1}(y, \tau)\right)} \leq c\left(\frac{\left|\mathfrak{C} \cap \mathcal{C}_{1}(y, \tau)\right|}{\left|\mathcal{C}_{1}(y, \tau)\right|}\right)^{\tau_{1}}
$$

To estimate the right-hand side, we note that

$$
\begin{aligned}
\frac{\left|\mathfrak{C} \cap \mathcal{C}_{1}(y, \tau)\right|}{\left|\mathcal{C}_{1}(y, \tau)\right|} & \leq c|\mathfrak{C}|=c\left|\left\{(x, t) \in Q: \mathcal{M}\left(|D u|^{2}\right)>\lambda_{1}^{2}\right\}\right| \\
& \leq c \int_{Q} \mathcal{M}\left(|D u|^{2}\right) d x d t \leq c \int_{Q}|D u|^{2} d x d t \\
& \leq c \int_{Q}|\mathbf{F}(x, t)|^{2} d x d t \leq \frac{c|Q|}{\omega(Q)^{\frac{2}{p}}}\left(\int_{Q}|\mathbf{F}(x, t)|^{2 \frac{p}{2}} \omega(x, t) d x d t\right)^{\frac{2}{p}} \\
& \leq c \delta^{2}
\end{aligned}
$$

for almost all $(y, \tau) \in \mathfrak{C}$, where we have used (2.6). Taking $\delta$ small enough, we get by $(2.9)$ that

$$
\Theta(1)=\frac{\omega\left(\mathfrak{C} \cap \mathcal{C}_{1}(y, \tau)\right)}{\omega\left(\mathcal{C}_{1}(y, \tau)\right)} \leq c \delta^{2 \tau_{1}}<\varepsilon,
$$

which ensures the validity of (4.6). Therefore Lemma 4.4 gives

$$
\begin{aligned}
\mathcal{S}:= & \sum_{k \geq 1} \lambda_{1}^{2 k \frac{p}{2}} \omega\left(\left\{(x, t) \in Q: \mathcal{M}\left(|D u|^{2}\right)>\lambda_{1}^{2 k}\right\}\right) \\
\leq & \sum_{k \geq 1} \lambda_{1}^{k p} \varepsilon_{1}^{k} \omega\left(\left\{(x, t) \in Q: \mathcal{M}\left(|D u|^{2}\right)>1\right\}\right) \\
& +\sum_{k \geq 1} \sum_{i=1}^{k} \lambda_{1}^{k p} \varepsilon_{1}^{i} \omega\left(\left\{(x, t) \in Q: \mathcal{M}\left(|\mathbf{F}|^{2}\right)>\delta^{2} \lambda_{1}^{2(k-i)}\right\}\right) \\
\leq & \sum_{k \geq 1}\left(\lambda_{1}^{p} \varepsilon_{1}\right)^{k} \omega(Q) \\
& +\sum_{i \geq 1}\left(\lambda_{1}^{p} \varepsilon_{1}\right)^{i} \sum_{k \geq i} \lambda_{1}^{p(k-i)} \omega\left(\left\{(x, t) \in Q: \mathcal{M}\left(|\mathbf{F}|^{2}\right)>\delta^{2} \lambda_{1}^{2(k-i)}\right\}\right) \\
\leq & \omega(Q) \sum_{k \geq 1}\left(\lambda_{1}^{p} \varepsilon_{1}\right)^{k}+\mathcal{S}^{\prime} .
\end{aligned}
$$


Let us note that

$$
\begin{aligned}
& \sum_{k \geq i} \lambda_{1}^{p(k-i)} \omega\left(\left\{(x, t) \in Q: \mathcal{M}\left(|\mathbf{F}|^{2}\right)>\delta^{2} \lambda_{1}^{2(k-i)}\right\}\right) \\
& =\sum_{k \geq i}\left(\lambda_{1}^{2(k-i)}\right)^{\frac{p}{2}} \omega\left(\left\{(x, t) \in Q: \mathcal{M}\left(\frac{|\mathbf{F}|^{2}}{\delta^{2}}\right)>\lambda_{1}^{2(k-i)}\right\}\right) \\
& \leq C\left\|\frac{|\mathbf{F}|^{2}}{\delta^{2}}\right\|_{L_{\omega}^{\frac{p}{2}}(Q)}^{\frac{p}{2}}=\frac{c}{\delta^{p}}\left\||\mathbf{F}|^{2}\right\|_{L_{\omega}^{\frac{p}{2}}(Q)}^{\frac{p}{2}} \leq c,
\end{aligned}
$$

where (4.8) has been used in the last estimate. Hence $\mathcal{S}^{\prime} \leq c \sum_{i \geq 1}\left(\lambda_{1}^{p} \varepsilon_{1}\right)^{i}$.

Taking $\varepsilon$ small enough in a way that $\lambda_{1}^{p} \varepsilon_{1}<1$, and consequently also $\delta$, we get $\mathcal{S}<\infty$ which gives

$$
\left\|\mathcal{M}\left(|D u|^{2}\right)\right\|_{L_{\omega}^{\frac{p}{2}}(Q)} \leq c(\omega(Q)+\mathcal{S})<\infty
$$

This way, Lemmas 2.2 and 4.5 imply

$$
\|D u\|_{L_{\omega}^{p}(Q)} \leq c\left(\|\mathbf{F}\|_{L_{\omega}^{p}(Q)}+\omega(Q)\right)
$$

and the desired estimate which completes the proof of Theorem 3.2 follows by the Banach inverse mapping theorem.

\section{Morrey regularity of the gradient}

A direct consequence of Theorem 3.2 is the Morrey regularity of the spatial gradient of the weak solution to the problem (1.1). Recall that the Morrey spaces $L^{q, \lambda}(Q)$ with $q>1$ and $\lambda \in(0, n+2)$ consist of all measurable functions $f \in L^{q}(Q)$ for which the following norm is finite

$$
\|f\|_{L^{q, \lambda}(Q)}=\left(\sup _{\substack{(y, \tau) \in Q \\ 0<r<\operatorname{diam} Q}} \frac{1}{r^{\lambda}} \int_{\mathcal{E}_{r}(y, \tau) \cap Q}|f(x, t)|^{q} d x d t\right)^{1 / q}<\infty
$$

where $\mathcal{E}_{r}(y, \tau)$ is any ellipsoid with radius $r$ and centered at $(y, \tau) \in Q$.

Theorem 5.1. Under the assumptions of Theorem 3.2, suppose in addition that $\mathbf{F} \in L^{p, \lambda}$ with $p>2$ and $\lambda \in(0, n+2)$. Then there exists a small positive constant $\delta=\delta(n, L, \nu, p, \lambda, Q)$ such that if the couple $(\mathbf{a}, \Omega)$ is $(\delta, R)$-vanishing of co-dimension 1 , then the spatial gradient $D u$ of the weak solution $u$ to the problem (1.1) belongs to $L^{p, \lambda}(Q)$ and satisfies the estimate

$$
\|D u\|_{L^{p, \lambda}(Q)} \leq c\|\mathbf{F}\|_{L^{p, \lambda}(Q)}
$$

with a constant $c$ independent of $u$ and $\mathbf{F}$.

Proof. Suppose that $\mathbf{F}: Q \rightarrow \mathbf{R}^{n}$ is extended as zero to the whole $\mathbf{R}^{n+1}$. Fix a point $\left(x_{0}, t_{0}\right) \in Q, r>0$, and consider the ellipsoid $\mathcal{E}_{r}\left(x_{0}, t_{0}\right)$ with a characteristic function $\chi_{\mathcal{E}_{r}\left(x_{0}, t_{0}\right)}$ and maximal function $\mathcal{M} \chi_{\mathcal{E}_{r}\left(x_{0}, t_{0}\right)}(x, t)$. It is proved [9, Proposition 2] (see also [29, Proposition IX.3.3]) that

$$
\left(\mathcal{M} \chi_{\mathcal{E}_{\left(x_{0}, t_{0}\right)}}\right)^{\sigma} \in A_{1} \quad \text { when } \quad 0 \leq \sigma<1 .
$$

Hence, by the definition (2.5) of an $A_{1}$-weight, we get

$$
\frac{1}{\left|\mathcal{E}_{r}\left(x_{0}, t_{0}\right)\right|} \int_{\mathcal{E}_{r}\left(x_{0}, t_{0}\right)}\left(\mathcal{M} \chi_{\mathcal{E}_{r}\left(x_{0}, t_{0}\right)}(x, t)\right)^{\sigma} d x d t \leq A \underset{\mathcal{E}_{r}\left(x_{0}, t_{0}\right)}{\operatorname{essinf}}\left(\mathcal{M} \chi_{\mathcal{E}_{r}\left(x_{0}, t_{0}\right)}\right)^{\sigma} .
$$


Because of the increasing property of the $A_{q^{-}}$classes we have $\left(\mathcal{M} \chi_{\mathcal{E}_{r}\left(x_{0}, t_{0}\right)}\right)^{\sigma} \in A_{\frac{p}{2}}$ for each $p>2$ with a bound $\left[\left(\mathcal{M} \chi_{\mathcal{E}_{r}\left(x_{0}, t_{0}\right)}\right)^{\sigma}\right]_{\frac{p}{2}} \leq A$ depending only on $n, p$ and $\sigma$. Therefore, applying the result of Theorem 3.2, we obtain

$$
\begin{aligned}
I & =\int_{\mathcal{E}_{r}\left(x_{0}, t_{0}\right) \cap Q}|D u|^{p} d x d t=\int_{Q}|D u|^{p}\left(\chi_{\mathcal{E}_{r}\left(x_{0}, t_{0}\right)}\right)^{\sigma} d x d t \\
& \leq \int_{Q}|D u|^{p}\left(\mathcal{M} \chi_{\mathcal{E}_{r}\left(x_{0}, t_{0}\right)}\right)^{\sigma} d x d t \leq c \int_{Q}|\mathbf{F}(x, t)|^{p}\left(\mathcal{M} \chi_{\mathcal{E}_{r}\left(x_{0}, t_{0}\right)}\right)^{\sigma} d x d t \\
& =c \int_{\mathbf{R}^{n+1}}|\mathbf{F}(x, t)|^{p}\left(\mathcal{M} \chi_{\mathcal{E}_{r}\left(x_{0}, t_{0}\right)}\right)^{\sigma} d x d t .
\end{aligned}
$$

Employing the dyadic decomposition of $\mathbf{R}^{n+1}$ related to $\mathcal{E}_{r}\left(x_{0}, t_{0}\right)$,

$$
\mathbf{R}^{n+1}=\mathcal{E}_{2 r}\left(x_{0}, t_{0}\right) \cup\left(\bigcup_{k=1}^{\infty} \mathcal{E}_{2^{k+1} r}\left(x_{0}, t_{0}\right) \backslash \mathcal{E}_{2^{k} r}\left(x_{0}, t_{0}\right)\right),
$$

the last bound becomes

$$
\begin{aligned}
I \leq & c\left(\int_{\mathcal{E}_{2 r}\left(x_{0}, t_{0}\right)}|\mathbf{F}(x, t)|^{p}\left(\mathcal{M} \chi_{\mathcal{E}_{r}\left(x_{0}, t_{0}\right)}\right)^{\sigma} d x d t\right. \\
& \left.\quad+\sum_{k=1}^{\infty} \int_{\mathcal{E}_{2^{k+1_{r}}}\left(x_{0}, t_{0}\right) \backslash \mathcal{E}_{2^{k_{r}}}\left(x_{0}, t_{0}\right)}|\mathbf{F}(x, t)|^{p}\left(\mathcal{M} \chi_{\mathcal{E}_{r}\left(x_{0}, t_{0}\right)}\right)^{\sigma} d x d t\right) \\
= & I_{0}+\sum_{k=1}^{\infty} I_{k} .
\end{aligned}
$$

Let us estimate now the maximal function of $\chi_{\mathcal{E}_{r}\left(x_{0}, t_{0}\right)}$. It follows by the definition that

$$
\begin{aligned}
\mathcal{M} \chi_{\mathcal{E}_{r}\left(x_{0}, t_{0}\right)}(y, \tau) & =\sup _{\mathcal{E}_{\bar{r}}(y, \tau)} \frac{1}{\left|\mathcal{E}_{\bar{r}}(y, \tau)\right|} \int_{\mathcal{E}_{\bar{r}}(y, \tau)} \chi_{\mathcal{E}_{r}\left(x_{0}, t_{0}\right)}(x, t) d x d t \\
& =\sup _{\mathcal{E}_{\bar{r}}(y, \tau)} \frac{\left|\mathcal{E}_{\bar{r}}(y, \tau) \cap \mathcal{E}_{r}\left(x_{0}, t_{0}\right)\right|}{\left|\mathcal{E}_{\bar{r}}(y, \tau)\right|}=\sup _{\bar{r}} \frac{r^{n+2}}{\bar{r} n+2}
\end{aligned}
$$

where $\mathcal{E}_{\bar{r}}(y, \tau)$ is an arbitrary ellipsoid centered at some point $(y, \tau) \in \mathbf{R}^{n+1}$.

If $(y, \tau) \in \mathcal{E}_{r}\left(x_{0}, t_{0}\right)$, then $\mathcal{M} \chi_{\mathcal{E}_{r}\left(x_{0}, t_{0}\right)}(y, \tau)=1$. On the other hand, if $(y, \tau) \in$ $\mathcal{E}_{2 r}\left(x_{0}, t_{0}\right) \backslash \mathcal{E}_{r}\left(x_{0}, t_{0}\right)$, then

$$
\mathcal{M} \chi_{\mathcal{E}_{r}\left(x_{0}, t_{0}\right)}(y, \tau)=\frac{r^{n+2}}{(2 r)^{n+2}}=\frac{1}{2^{n+2}}<1 .
$$

Let $(y, \tau) \in \mathcal{E}_{2^{k+1} r}\left(x_{0}, t_{0}\right) \backslash \mathcal{E}_{2^{k} r}\left(x_{0}, t_{0}\right)$. We have

$$
\begin{aligned}
2^{k-1} r & \leq 2^{k} r-r \leq \rho\left(y-x_{0}, \tau-t_{0}\right)-r \leq \bar{r} \\
& \leq \rho\left(y-x_{0}, \tau-t_{0}\right)+r \leq 2^{k+1} r+r,
\end{aligned}
$$

and the maximal function is evaluated by

$$
\mathcal{M} \chi_{\mathcal{E}_{r}\left(x_{0}, t_{0}\right)}(y, \tau) \leq \frac{r^{n+2}}{\left(2^{k-1} r\right)^{n+2}}=\frac{1}{2^{(k-1)(n+2)}} .
$$


We are in a position now to estimate the terms $I_{k}, k=0,1, \ldots$ Namely,

$$
\begin{aligned}
I_{0} & \leq \int_{\mathcal{E}_{2 r}\left(x_{0}, t_{0}\right)}|\mathbf{F}(x, t)|^{p} d x d t \leq C(n) r^{\lambda}\|\mathbf{F}\|_{L^{p, \lambda}(Q)}^{p}, \\
I_{k} & \leq \frac{1}{2^{(k-1)(n+2) \sigma}} \int_{\mathcal{E}_{2^{k+1} \backslash \backslash \mathcal{E}_{2^{k} r}}\left(x_{0}, t_{0}\right)}|\mathbf{F}(x, t)|^{p} d x d t \\
& \leq \frac{1}{2^{(k-1)(n+2) \sigma}} \int_{\mathcal{E}_{2^{k+1} r}\left(x_{0}, t_{0}\right)}|\mathbf{F}(x, t)|^{p} d x d t \\
& =\frac{\left(2^{k+1} r\right)^{\lambda}}{2^{(k-1)(n+2) \sigma}} \frac{1}{\left(2^{k+1} r\right)^{\lambda}} \int_{\mathcal{E}_{2^{k+1} r}}|\mathbf{F}(x, t)|^{p} d x d t \\
& \leq C(n, \sigma, \lambda) 2^{k(\lambda-(n+2) \sigma)} r^{\lambda}\|\mathbf{F}\|_{L^{p, \lambda}(Q)}^{p},
\end{aligned}
$$

which leads to

$$
\int_{\mathcal{E}_{r}\left(x_{0}, t_{0}\right) \cap Q}|D u|^{p} d x d t \leq C\left(1+\sum_{k=1}^{\infty} 2^{\lambda-(n+2) \sigma}\right) r^{\lambda}\|\mathbf{F}\|_{L^{p, \lambda}(Q)}^{p} .
$$

At this point we choose $\sigma \in\left(\frac{\lambda}{n+2}, 1\right)$ in order to ensure convergence of the series above. To get the desired estimate (5.1), it remains to divide the both sides by $r^{\lambda}$ and to take supremum with respect to $0<r<\operatorname{diam} Q$ and $\left(x_{0}, t_{0}\right) \in Q$.

\section{Linear parabolic systems in divergence form}

The previous results could be easily extended to the case of non-homogeneous parabolic systems in divergence form

$$
\begin{cases}u_{t}^{i}-D_{\alpha}\left(a_{i j}^{\alpha \beta}(x, t) D_{\beta} u^{j}\right)=D_{\alpha} f_{\alpha}^{i}(x, t) & \text { in } Q, \\ u^{i}(x, t)=0 & \text { on } \partial_{P} Q,\end{cases}
$$

for $i=1, \ldots, m$.

The tensor matrix of the coefficients

$$
\mathbf{A}=\left\{a_{i j}^{\alpha \beta}\right\}: \mathbf{R}^{n+1} \rightarrow \mathbf{R}^{m n \times m n}
$$

is assumed to be uniformly bounded and uniformly parabolic, namely, we suppose that there exists positive constants $L$ and $\nu$ such that

$$
\|\mathbf{A}\|_{L^{\infty}\left(\mathbf{R}^{n+1}, \mathbf{R}^{m n \times m n}\right)} \leq L, \quad a_{i j}^{\alpha \beta}(x, t) \xi_{\alpha}^{i} \xi_{\beta}^{j} \geq \nu|\xi|^{2}
$$

for all matrices $\xi \in \mathcal{M}^{m \times n}$ and for almost every $(x, t) \in \mathbf{R}^{n+1}$.

When the non-homogeneous term $\mathbf{F}=\left\{f_{\alpha}^{i}\right\}$ belongs to $L^{2}\left(Q, \mathbf{R}^{m n}\right)$, the CauchyDirichlet problem (6.1) has a unique weak solution $\mathbf{u}=\left(u_{1}, \ldots, u_{m}\right)$ with the standard $L^{2}$-estimate

$$
\|D \mathbf{u}\|_{L^{2}\left(Q, \mathbf{R}^{n m}\right)} \leq c\|\mathbf{F}\|_{L^{2}\left(Q, \mathbf{R}^{n m}\right)},
$$

where $c$ is a positive constant depending only on $n, m, L, \nu$ and $|Q|$. In particular, the weak solution of (6.1) belongs to

$$
H^{\frac{1}{2}}\left(0, T ; L^{2}\left(\Omega, \mathbf{R}^{m}\right)\right) \cap L^{2}\left(0, T ; H_{0}^{1}\left(\Omega, \mathbf{R}^{m}\right)\right),
$$

and satisfies the estimate

$$
\|\mathbf{u}\|_{H^{\frac{1}{2}}\left(0, T ; L^{2}\left(\Omega, \mathbf{R}^{m}\right)\right) \cap L^{2}\left(0, T ; H_{0}^{1}\left(\Omega, \mathbf{R}^{m}\right)\right)}+\|D \mathbf{u}\|_{L^{2}\left(Q, \mathbf{R}^{m n}\right)} \leq c\|\mathbf{F}\|_{L^{2}\left(Q, \mathbf{R}^{m n}\right)},
$$

where the constant $c$ is independent of $\mathbf{u}$ and $\mathbf{F}$. 
The proofs given in Sections 4 and 5 apply also to the weak solutions of the system (6.1). That is why, we shall restrict ourselves only to announce the corresponding regularity results.

Theorem 6.1. Assume $(6.2)$ and let $p \in(2, \infty)$ and $\omega \in A_{\frac{p}{2}}$. There exists a small positive constant $\delta=\delta(n, m, L, \nu, p, \omega)$ such that if the couple $(\mathbf{A}, \Omega)$ is $(\delta, R)$ vanishing of co-dimension 1 and $\mathbf{F} \in L_{\omega}^{p}\left(Q, \mathbf{R}^{m n}\right)$, then the spatial gradient $D \mathbf{u}$ of the weak solution $\mathbf{u}$ to $(6.1)$ lies in $L_{\omega}^{p}\left(Q, \mathbf{R}^{m n}\right)$ and satisfies the estimate

$$
\|D \mathbf{u}\|_{L_{\omega}^{p}\left(Q, \mathbf{R}^{m n}\right)} \leq c\|\mathbf{F}\|_{L_{\omega}^{p}\left(Q, \mathbf{R}^{m n}\right)},
$$

with a constant $c$ independent of $\mathbf{u}$ and $\mathbf{F}$.

Corollary 6.2. Under the assumptions of Theorem 6.1, there is a small positive constant $\delta=\delta(n, m, L, \nu, p, \lambda)$ such that if the couple $(\mathbf{A}, \Omega)$ is $(\delta, R)$-vanishing of co-dimension 1 and $\mathbf{F} \in L^{p, \lambda}\left(Q, \mathbf{R}^{m n}\right)$ with $p \in(2, \infty)$ and $\lambda \in(0, n+2)$, then $D \mathbf{u} \in L^{p, \lambda}\left(Q, \mathbf{R}^{m n}\right)$ and

$$
\|D \mathbf{u}\|_{L^{p, \lambda}\left(Q, \mathbf{R}^{m n}\right)} \leq c\|\mathbf{F}\|_{L^{p, \lambda}\left(Q, \mathbf{R}^{m n}\right)}
$$

with a constant $c$ independent of $\mathbf{u}$ and $\mathbf{F}$.

\section{References}

[1] Byun, S.-S.: Parabolic equations with BMO coefficients in Lipschitz domains. - J. Differential Equations 209:2, 2005, 229-265.

[2] Byun, S.-S.: Optimal $W^{1, p}$ regularity theory for parabolic equations in divergence form. - J. Evol. Equ. 7:3, 2007, 415-428.

[3] Byun, S.-S., and D.K. Palagachev: Weighted $L^{p}$-estimates for elliptic equations with measurable coefficients in nonsmooth domains, - Potential Anal. 41:1, 2014, 51-79.

[4] Byun, S.-S., and D. K. Palagachev: Morrey regularity of solutions to quasilinear elliptic equations over Reifenberg flat domains. - Calc. Var. Partial Differential Equations 49:1-2, 2014, 37-76.

[5] Byun, S.-S., D. K. Palagachev, and L. Wang: Parabolic systems with measurable coefficients in Reifenberg domains. - Int. Math. Res. Not. IMRN 2013:13, 2013, 3053-3086.

[6] Byun, S.-S., and L. Wang: Parabolic equations in Reifenberg domains. - Arch. Ration. Mech. Anal. 176:2, 2005, 271-301.

[7] ByUn, S., and L. WANG: $L^{p}$-regularity for fourth order parabolic systems with measurable coefficients. - Math. Z. 272:1-2, 2012, 515-530.

[8] Chipot, M., D. Kinderlehrer, and G. Vergara-Caffarelli: Smoothness of linear laminates. - Arch. Ration. Mech. Anal. 96:1, 1986, 81-96.

[9] Coifman, R. R., and R. Rochberg: Another characterization of BMO. - Proc. Amer. Math. Soc. $79: 2,1980,249-254$.

[10] David, G., and T. Toro: Reifenberg parameterizations for sets with holes. - Mem. Amer. Math. Soc. 215:1012, 2012.

[11] Dong, H.: Gradient estimates for parabolic and elliptic systems from linear laminates. - Arch. Ration. Mech. Anal. 205:1, 2012, 119-149.

[12] Dong, H., and D. KIm: Higher order elliptic and parabolic systems with variably partially BMO coefficients in regular and irregular domains. - J. Funct. Anal. 261:11, 2011, 3279-3327.

[13] Elschner, J., J. Rehberg, and G. Schmidt: Optimal regularity for elliptic transmission problems including $C^{1}$ interfaces. - Interfaces Free Bound. 9:2, 2007, 233-252.

[14] Fabes, E. B., and N. M. Rivière: Singular integrals with mixed homogeneity. - Studia Math. $27,1966,19-38$. 
[15] García-Cuerva, J., and J. L. Rubio de Francia: Weighted norm inequalities and related topics. - North-Holland Mathematics Studies 116, Notas de Matematica [Mathematical Notes] 104, North-Holland Publishing Co., Amsterdam, 1985.

[16] Guliyev, V.S., and L. G. Softova: Generalized Morrey regularity for parabolic equations with discontinous data. - Proc. Edinb. Math. Soc. (2) 58:1, 2015, 199-218.

[17] Kenig, C., and T. Toro: Poisson kernel characterization of Reifenberg flat chord arc domains. - Ann. Sci. École Norm. Sup. (4) 36:3, 2003, 323-401.

[18] Lemenant, A., E. Milakis, and L. V. Spinolo: On the extension property of Reifenberg-flat domains. - Ann. Acad. Sci. Fenn. Math. 39:1, 2014, 51-71.

[19] Li, Y., and L. Nirenberg: Estimates for elliptic systems from composite material. - Comm. Pure Appl. Math. 56:7, 2003, 892-925.

[20] Li, Y., and M. Vogelius: Gradient estimates for solutions to divergence form elliptic equations with discontinuous coefficients, - Arch. Ration. Mech. Anal. 153:2, 2000, 91-151.

[21] Mengesha, T., and N. C. Phuc: Weighted and regularity estimates for nonlinear equations on Reifenberg flat domains. - J. Differential Equations 250:5, 2011, 2485-2507.

[22] Muckenhoupt, B.: Weighted norm inequalities for the Hardy maximal function. - Trans. Amer. Math. Soc. 165, 1972, 207-226.

[23] Reifenberg, E. R.: Solution of the Plateau problem for $m$-dimensional surfaces of varying topological type. - Acta Math. 104, 1960, 1-92.

[24] Palagachev, D. K., and L. G. Softova: Quasilinear divergence form parabolic equations in Reifenberg flat domains. - Discrete Contin. Dyn. Syst. 31:4, 2011, 1397-1410.

[25] Softova, L. G.: Singular integrals and commutators in generalized Morrey spaces. - Acta Math. Sin. (Engl. Ser.) 22:3, 2006, 757-766.

[26] Softova, L. G.: Morrey-type regularity of solutions to parabolic problems with discontinuous data. - Manuscripta Math. 136:3-4, 2011, 365-382.

[27] Softova, L. G.: Parabolic oblique derivative problem with discontinuous coefficients in generalized Morrey spaces. - Ric. Mat. 62:2, 2013, 265-278.

[28] Stein, E.: Harmonic analysis: real-variable methods, orthogonality and oscillatory integrals. Princeton Math. Ser. 43, Monographs in Harmonic Analysis III, Princeton Univ. Press, Princeton, NJ, 1993.

[29] Torchinsky, A.: Real variable methods in harmonic analysis. - Pure and Applied Mathematics 123, Academic Press, Inc., Orlando, FL, 1986.

[30] Toro, T.: Doubling and flatness: geometry of measures. - Notices Amer. Math. Soc. 44:9, 1997, 1087-1094. 